\title{
Hardware Implementation of MISO on Orthogonal Frequency Division Multiplexing Platform with the Help of Alamouti Algorithm
}

\author{
Seetaiah Kilaru
}

\begin{abstract}
Many software based OFDM techniques were proposed from last half decade to improve the performance of the system. This paper tried to implement the same with Hardware implementation. We created Hardware based MISO platform with OFDM. We implemented Alamouti algorithm on this test bed. The test bed is implemented with the help of Field Programmable Gate Array (FPGA). The test bed is functionalized with the help of FPGA through Xilinx based system generator for DSP. In this paper we considered the $2 \times 1$ MISO implementation with Alamouti algorithm. The simulation results showed that BER and SNR are considerably high for MISO than SISO. The results also proved that proposed OFDM based Alamouti implementation for MISO is excellent in all performance criterions.
\end{abstract}

Keywords-MISO, SISO, FPGA, Xilinx, BER, SNR, Alamouti, LTE

\section{INTRODUCTION}

$\mathbf{T}$ HE Wireless systems are playing important role in the society with respect to information transfer from one system to other system with high data rates. In future, this data rate speeds need to be improved and hence many broadband wireless networks are introducing Orthogonal Frequency Division Multiplexing (OFDM). With this, network operators are expecting to achieve high data rates. The recent advanced technologies like Long Term Evolution (LTE) and its Advanced (LTE-A) version is also using OFDM [9,13]. The efficiency of the service of a broadband wireless networks depends on the use of multiple antennas at transmitter/receiver. Multiple Input Multiple Output (MIMO) is one such technology to improve the performance of the system and also to mitigate the channel fading. By incorporating multiple antennas at both transmitter and receiver, we can improve the capacity of the system [22].

With MIMO, we can achieve antenna diversity and also we can increase multiplexing gain. This paper mainly focused on the antenna diversity technique. In $[6,10]$, the authors proposed space time frequency coding schemes to implement MIMO at the transmitter with multiple antennas and the design of receiver with efficient signal processing. In [27], they proposed space time frequency coding with the combination of OFDM to achieve MIMO optimization.

We come across various test beds in the implementation of OFDM systems based on Software Defined Radio (SDR). One

S. Kilaru is working in Electronics and Communication Engineering department, BVRIT Hyderabad College of Engineering for Women, Hyderabad, Telangana, INDIA (e-mail: dr.seetaiah@gmail.com) test bed which gathered our attention is the implementation of OFDM modulation with synchronization technique and error controlling phenomenon as explained in [16]. In [1], to transfer OFDM signals with Quadrature Phase Shift Keying (QPSK), they used GNU radio. With this analysis they found packet received radio of the system to analyze Quality of Service (QoS) issues. In [21], they proposed various possible WARP platforms to implement MIMO. In [17], the authors implemented OFDM system with Alamouti block code. In this work, they mainly focused on $2 \times 1$ (transmitting antennas $=2$ and receiving antenna $=1$ ) Multiple Input Single Output (MISO). In [7, 21], the authors designed high level models using Xilinx operated FPGA system for 802.11a and 802.16 modulators. In [13], they made an attempt to implement LTE communication standards. In this paper, we are presenting the SDR system in the implantation of both pre-coding and postcoding. We considered the Single Input Single Output (SISO) platform to design reconfigurable OFDM transceiver. This transceiver interfaces the Up/Down converter to the Radio Frequency unit. Here, we are considering this platform to implement the Alamouti techniques.

Frame synchronization is very important at the receiver side and it will determine the channel estimation parameters and provide channel equalization. The information or state of the channel is required for pre and post processing algorithms. Hence, we can conclude that, for a better optimization receiver should have 2 features, one is synchronization and the other is channel estimation. Synchronization should be achieved between the received RF carrier (RF-C) and local oscillator (LO). This will happen only when the receiver approximate frame arrival time and frequency off set (between LO and RFC). Like other existed systems, $802.11 \mathrm{a} / \mathrm{g}$, this system does not train any sequence to achieve Time Domain Synchronization (TDS).In general an OFDM symbol will carry information about both coded data (Information) and control data. The receiver has the prior knowledge about the transmitted value of carrier in symbol. The coded data should be codified in such a way that the system is reconfigurable. To achieve this reconfiguration, an OFDM symbol is coded in the order of data, zeros and pilots. In [32], the authors attempted to design optimum SISO-OFDM chain based on LTE. In our proposed analysis, we consider this arrangement as a primary reference. We introduced two transmitting antennas are placed instead of single antenna as implemented in SISO.

The proposed system aims at designing of efficient reconfigurable OFDM receiver with the help of FPGA. With this design, our objective is to achieve Time Synchronization, channel estimation and optimum Zero Force (ZF) 
equalizations. The proposed system is a high level design with the help of simulink, Matlab, FPGA and Xilinx. In the next sections, we explained OFDM transceiver implementation with the help of FPGA. We also explained the modified MISO from existed SISO using Alamouti algorithm. Finally, we implemented test bed and shown some of the validation results.

\section{OFDM TRANSCEIVER}

The following section explains about the convention SISO OFDM implementation.

\section{A. Transmitter}

The figure 1 shows the basic architecture of OFDM transceiver. Here, we used M-QAM modulation. Transmitter should be in a way that the codified OFDM symbols should be assembled in a flexible way to transmit and each symbol has the data and pilots to the sub-carrier and are mapped in a codifying vector. These set of vectors will represents the symbol type. There are various symbol types may generated, as an example one for synchronization and other for only data carrier information.

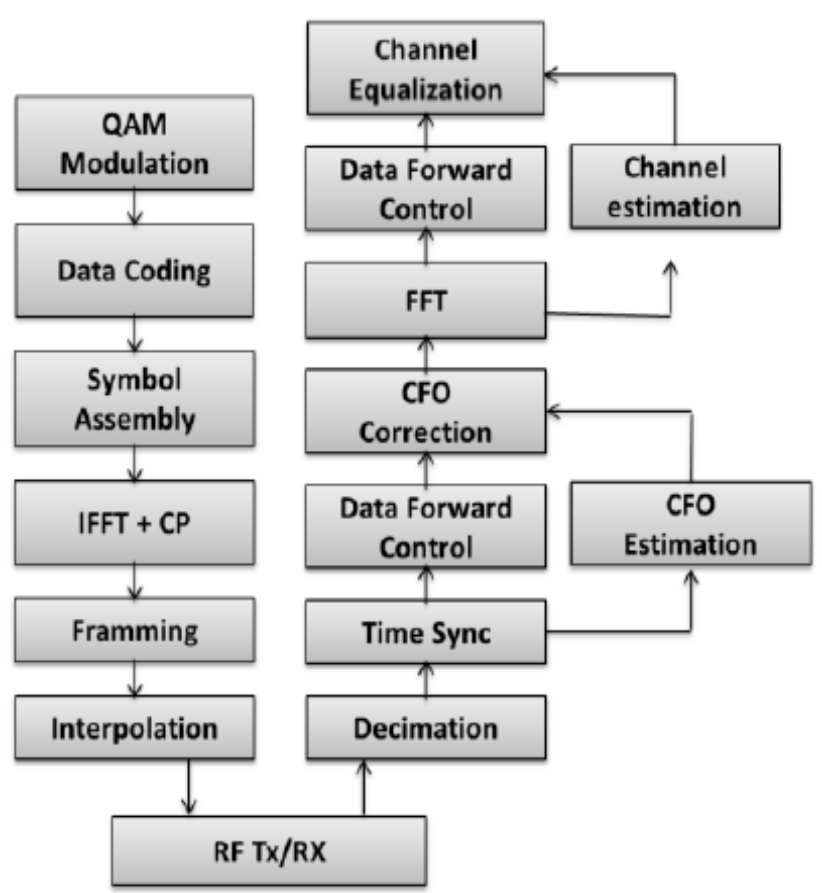

Fig. 1. OFDM Transceiver

The following figure 2 will give you overlook on implementation of flexibility in our system. Here, carrier index is given to ROM, where it has address of frequency carriers and be saved in a matrix form. the matrix has rows and columns, where rows represents subcarrier index type and columns have addresses and symbols. ROM codifies the available information in such a way that the specific carrier, data, pilots and zeros are in the proper order. In this total data, the least significant 10 bits has the information regarding frequency carrier index value.

We used 2 multiplexers here to observe how the sequence is formed with the attributes of data, pilots and zeros are placed

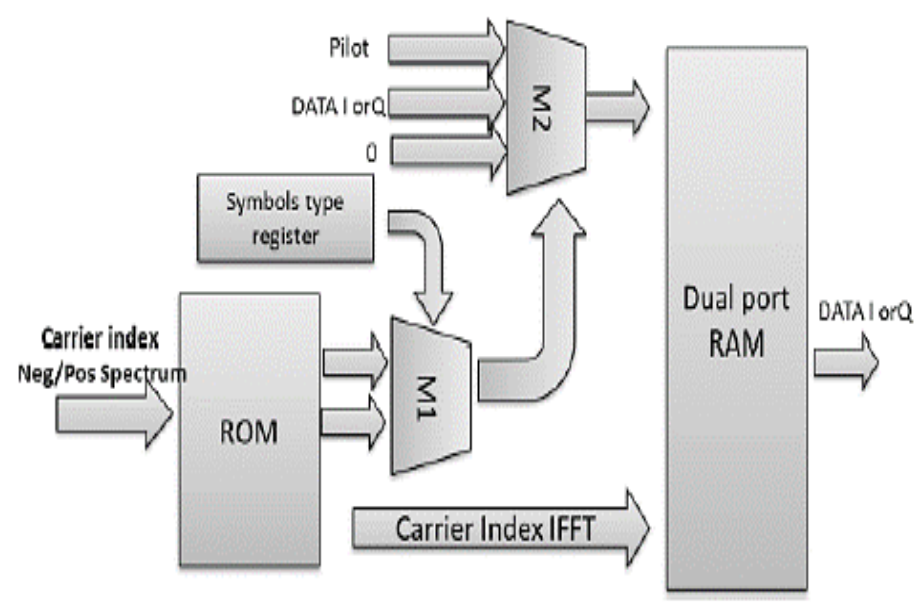

Fig. 2. Assembly block Diagram

in symbol. As we already told, symbols has many types, hence based on the type of symbol, multiplexer 1 will select 2 bits out of 4 bit column matrix.

$$
\underset{\text { data }}{\left[b_{13} b_{12}\right]\left[b_{11} b_{10}\right]}\left[b_{9} b_{8} \ldots . b_{2} b_{1} b_{0}\right]
$$

Selection of column depends on 2 factors, they are

- Sequence of symbol types in frame

- How symbols are stored in sequence register

From the figure 2, we can observe that the multiplexer 2 depends on multiplexer 1 output and then multiplexer 2 decide to multiplex data, pilots and zeros. This data placed in the right sequence to fill the symbol memory. This set up allows accommodating different types of symbols. In transmitter we are generating data by using Inverse Fast Fourier Transform (IFFT) of QAM symbols. This modulation scheme is implemented with 1024 sub-carriers.

\section{B. Receiver}

In MIMO, the receiver function is more complicated than the transmitted part. It involves with many functions includes synchronization of received signal, frame detection and error detection and correction. Now, we will explain how all these complicated tasks handled by our receiver with respect to above defined conditions.

\section{Time Domain Synchronization}

In wireless network operating system, transmitter and receiver has their own local oscillators and both are independent of each other. But, efficient demodulation requires synchronization. The received bits have the information of start bit, end bit and transmitted information. Now, the transmitted information should synchronize with the receiver with respect to time or frequency.

$$
r(k)=s(k-\tau) e^{\frac{j 2 \pi \varepsilon k}{N}}+n(k)
$$

Where $\varepsilon$ represents Carrier Frequency Offset (value to be normalized) and $\tau$ is the time which represents information about frame arrival time. $\mathrm{S}(\mathrm{k})$ id the total signal which is transmitted from the transmitter and $\mathrm{N}$ represents the total 
number of available samples. Here $\mathrm{k}$ value ranges from 0 to 1023 , i.e. 1024 sub carriers and is also called as a sample index of symbol and $\mathrm{n}(\mathrm{k})$ is the noise entered into the system. In this paper, we assumed additive white Gaussian noise. Moose in [2] and Beek in [28] proposed a simple model to calculate received signal at receiver. In [18], the authors introduced a robust algorithm with the method of preamble repetition.

In our paper also, we used the method of preamble repetition along with the help of Zadoff-Chu (ZC) sequences. This sequence used at frame starting point to make synchronization. $\mathrm{ZC}$ sequence has excellent autocorrelation properties and this technique is a part of 3GPP. Beek algorithm was used in our method due to low complexity and is shown in figure 3 .

The time domain synchronization is always a challenging task and can be handled with the help of an algorithm 3 steps. This proposed algorithm used the concept of Beek algorithm and the subsystem created fir its purpose and adapted to the frame pattern as shown in figure 4 is illustrated in figure 5. Beek used the correlation of $\mathrm{CP}$ with correlation with $\mathrm{CP}$ Delay. In this process, if we find out any repeated pattern, then the peak is observed and it indicates that the frame is arriving and the resultant phase difference between these two patterns will give carrier frequency offset.

This algorithm consists of 2 parts, i.e. in one part, we calculated energy term and in other part, we calculated correlation term. These two parameters are essential to calculate arrival time and frequency offset. The following equations will give information about energy term and correlation term.

$$
\begin{gathered}
\text { energy }=\frac{\rho}{2} \sum_{k=m}^{m+L+1}|r(k)|^{2}+|r(k+N)|^{2} \\
\text { correlation }=\frac{\rho}{2} \sum_{k=m}^{m+L+1}|r(k)| *|r(k+N)|
\end{gathered}
$$

Estimation of channel is an important task and also challenging task. With this, we can mitigate fading effects at the receiver also.

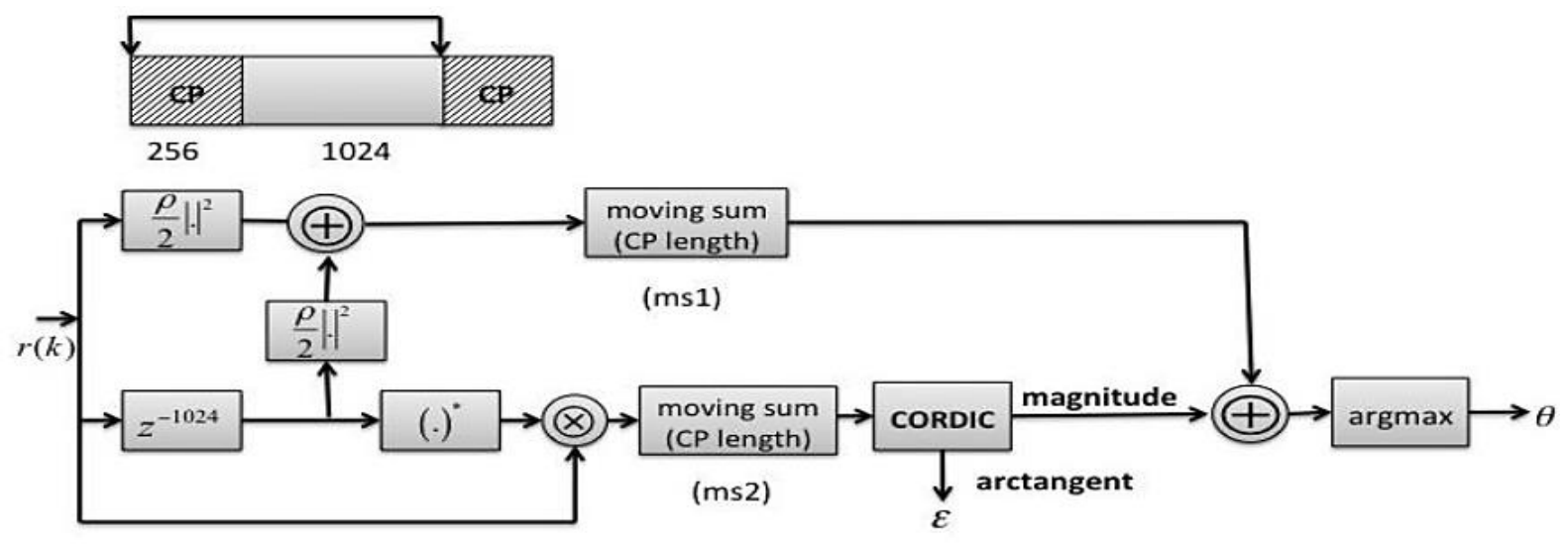

Fig. 3: Beek model for Synchronization

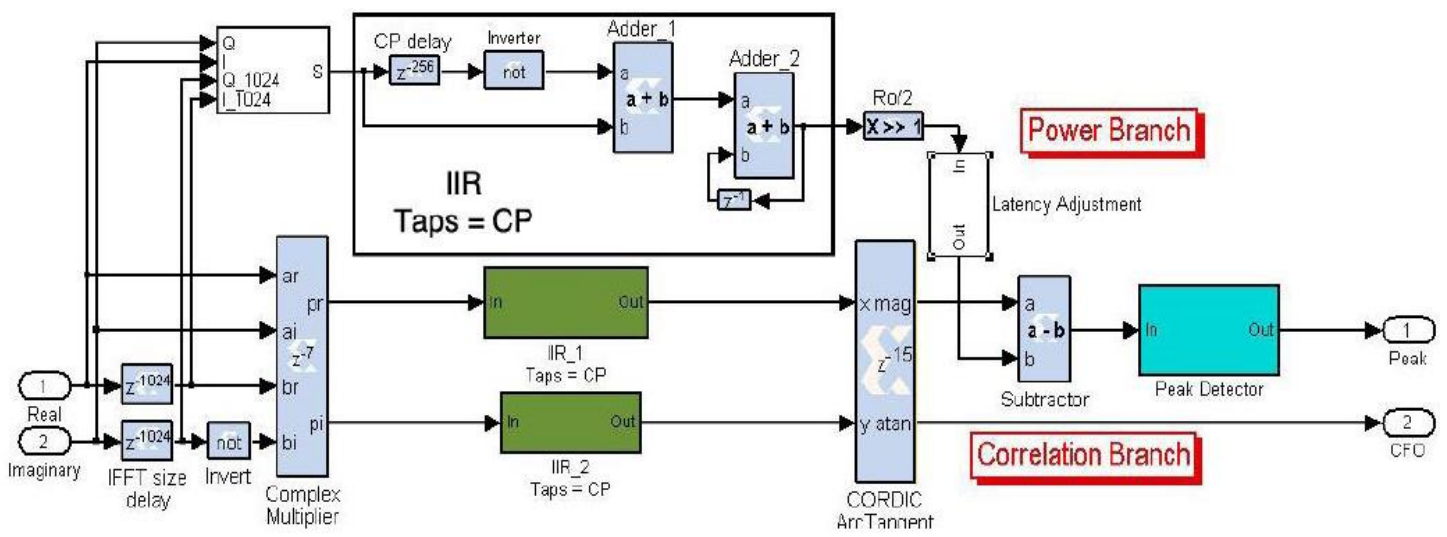

Fig. 4. Beek estimation with the help of XILINX system 


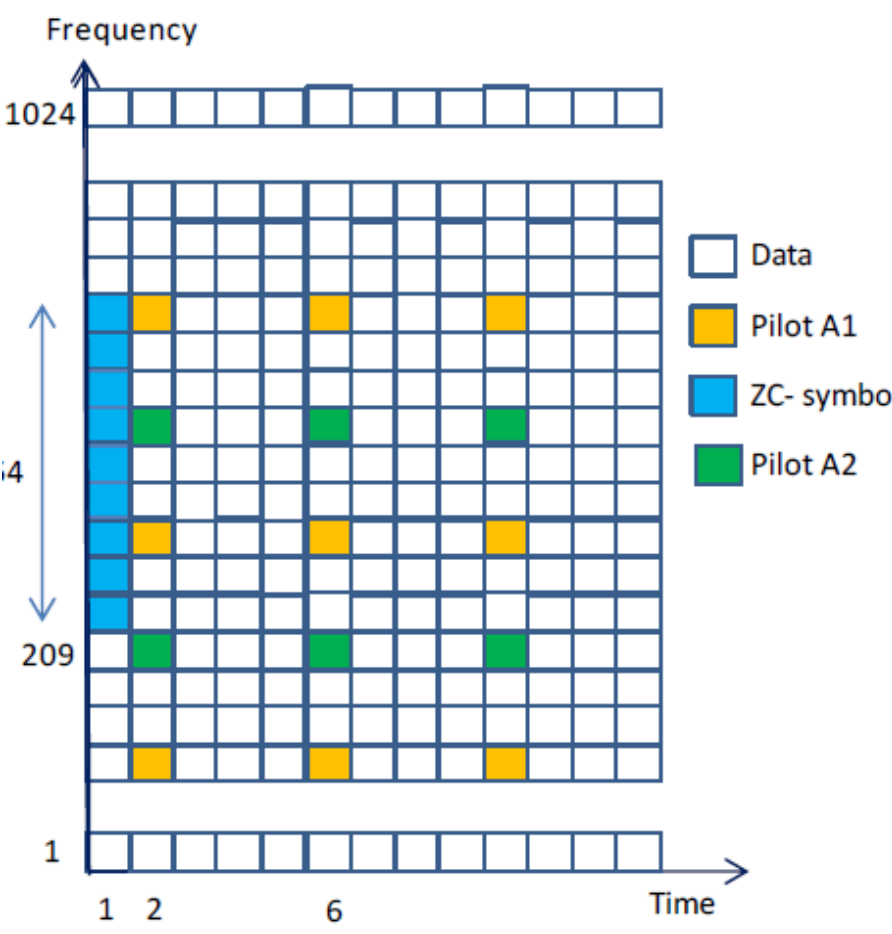

Fig. 5. Frame format of 2 antennas

Spectral efficiency of any OFDM system depends on the Channel State Information (CSI). The proper estimation of CSI depends on demodulation and decoding process. We should find the channel response at all available carrier frequencies in decoding process. The defined synchronization algorithm also defined the phase offset ambiguity. Channel estimator initially estimate frequency offset correction and the same can be eliminated in equalization process.

In this paper, we used common rectangular pilot pattern. We implemented the 12 symbol OFDM in which 1st, 5th and 9th symbols represents pilot sequence. As explained in $[8,12]$, to achieve Minimum Mean Square Error (MMSE), all existed pilot carried carriers should be equidistance from each other and also be equally power up (all existed sequences).

From the above analysis, we should note down that the consecutive pilots are separated by 6 sub-carriers. The frame structure is shown in figure 6 . In $[3,23,31]$, authors proposed the channel estimation algorithm. Extrapolate edge carriers as explained in $[19,30]$ are the complex issues which may rise in estimation. To overcome this problem, the proposed frame structure is defined with pilots at both edge sub-carriers.

In this paper, LS estimator algorithm [24] is used in the estimation of pilot subcarriers. This is the conventional channel estimator which does not take any advantage of correlation of channel across subcarriers in frequency. This also has the advantage of complexity reduction in implementation process. This requires only two simple mathematical operations such as inversion and multiplication. For kth pilot, the received subcarrier $\mathrm{p}(\mathrm{k})$ can be expressed as

$$
u(k)=s(k) h(k)+n(k)
$$

Here, $h(k)$ represents the channel values which is effecting the kth pilot sub-carrier. The output of the LS estimator can be given as

$$
\hat{\mathrm{h}}(k)=\frac{p(k)}{s(k)}=h(k)+\frac{n(k)}{s(k)}
$$

In [23] and some other works, the channel estimation output deals about both data subcarrier and pilot sub carrier. In case of our proposed method, it only estimates the information about pilot subcarriers. Now, we have to estimate the remaining sub carriers information i.e. data sub carriers. It can be done very easily by increasing the current channel estimation to the nearest pilots in frequency domain and if necessary in time domain also. In this paper, we used both time and frequency domains. This method can be only useful if the correlation of neighboring CFR is almost similar. That means, this is only an acceptable solution where the channel characteristics varies very slowly and where the delay spread is very limited. We used linear interpolation method with respect to frequency domain to design the transceiver. The similar model is implemented in [14, 29], they used primary order polynomial to define the line which can bifurcate the two neighboring points. This method is improvisation of [20]. If the designer focused on accuracy of the system, we should use the higher order systems as explained in [5, 11 and 25].

\section{MISO WITH ALAMOUTI ALGORITHM}

In this section, we explained the implementation of Alamouti algorithm. We implemented this algorithm with the help of SISO hardware. This platform is very useful to encode transmitted signals and also to decode the received signal. The proposed system requires both pre and post processing at both ends of the system. Antenna diversity is implemented with the help of Alamouti encoding and decoding. This implementation does not focus on higher data rates but focused on link robustness. This should be achieved without increasing the bandwidth of the system and also the power of the system. Here, we used 2 transmitting antennas and 1 receiving antennas. The system has gain of 2 with uncorrelated condition channel. The designed code at transmitter is a simple code generated with the help of spatial and time diversity. We used linear decoding at the receiver side with low complexity.

\section{A. Alamouti Transceiver}

The following figure 6 represents transmitter and receiver architectures. We used the same hardware components of SISO OFDM and additionally added Alamouti encoder and decoder blocks. We focused on MISO, so SISO converted to MISO by changing the position of transmitter after the encoder block. Channel estimation can be done with the help of pilots carried by OFDM symbols. These symbols should have in each branch and their respective pilot sub carriers are placed in non-coincident indexes. In each branch, the OFDM symbol, pilot occupied 6th position and between two pilots, zero is transmitted. In the next branch, we interchanged the positions of pilots and zeros. With this arrangement, we can estimate the channel at the receiver. Two different channels can be estimated with two different pilot sub carriers. 


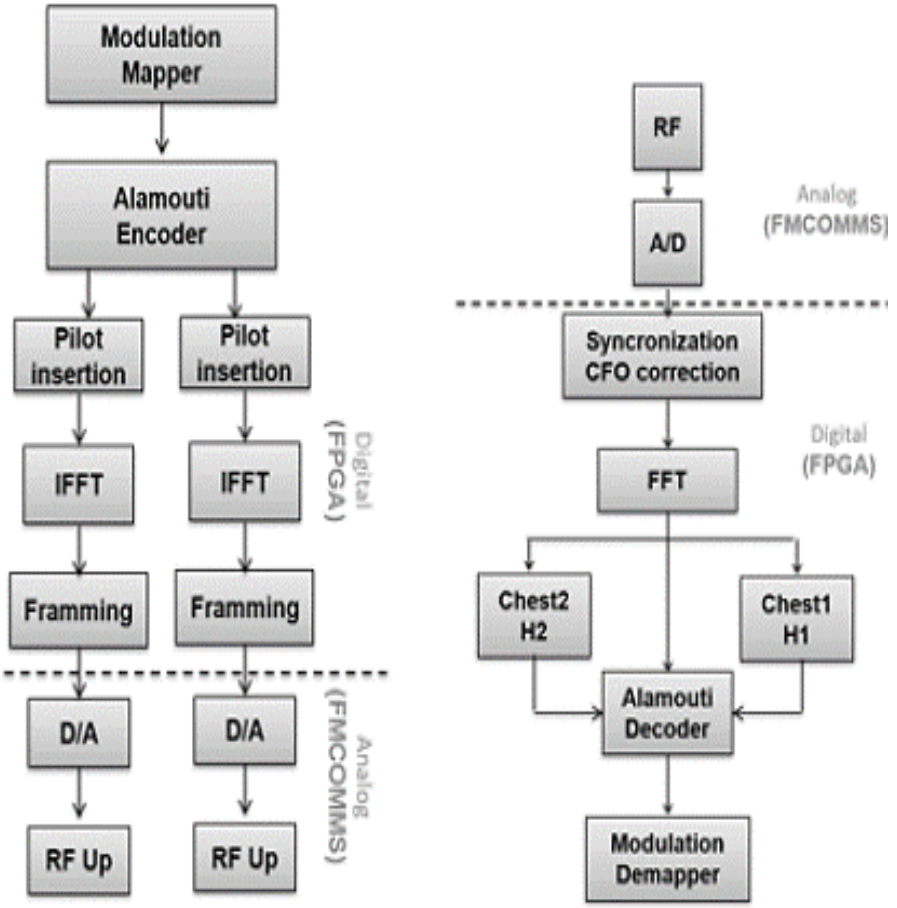

Fig. 6. Alamouti Transceiver

\section{B. Alamouti encoder/decoder}

The process of coding consists of 2 set of codes, they are code 1 and code 2 . Code 1 gives information about transmitter without any modifications and code 2 is Alamouti code. For the defined frequency $\mathrm{k}$, antenna 1 can define sequence sk and antenna 2 can define the sequence $s k+1$. For the frequency $\mathrm{k}+1$, antenna 1 can define the sequence in relate to the antenna 1 as - sk +1 and antenna 2 can define the sequence to the relate of antenna 1 code as sk*. This defined implementation needs data time sequence swapping between 2 symbols. This swapping can be performed with the help of following circuit as shown in figure 7 .

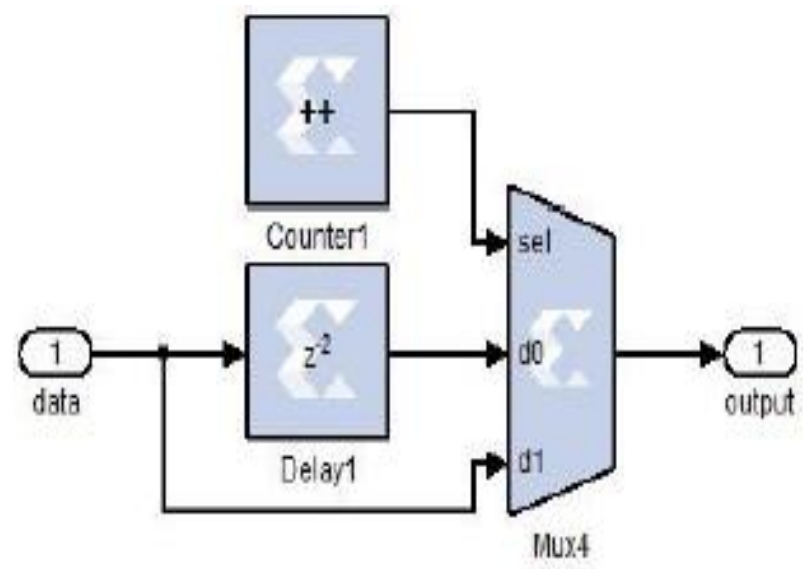

Fig. 7. Time sequence reversal process

Initially, we should create 2 sequences in such a way that one sequence to be in delay with other sequence. Then using multiplexer, we can perform sampling alternatively. With this time order of sequences can be achieved. Additionally, we need counter to count between zeros and $\mathrm{ON}$.

\section{Channel Estimation}

To decode, one should require channel estimation. Channel estimation can be done only with the help of transmission between antenna 1 and receiver and also between antenna 2 and receiver. We used QPSK modulation to resolve this problem. Antenna 1 should transmit symbols where the pilot subcarriers of antenna 2 are set to zero. We should observe relative index of two pilot subcarriers by two positions. This means that when antenna 1 is transmitting data, antenna 2 set to be null and follows vice-versa.

\section{NUMERICAL RESULTS}

For simulation analysis, we considered MISO SDR platform. In this we assumed 2 transmitting antennas and 1 receiving antenna. Alamouti algorithm is implemented on transmitter. We used Rayleigh channel model to model the channel between transmitter and receiver. The channel modeling analysis is done with the help of simulink. We used MATLAB to find out the filter coefficients. Initially we considered Additive White Gaussian Noise (AWGN) spread over the channel and we calculated Bit Error Rate (BER). In next scenario, we considered SISO with Rayleigh channel modeling. In other scenario, we considered 2 transmitting antennas transmitting to one receiving antennas with Rayleigh channel model. The following figure 8 will give you outlook on MISO Alamouti comparison with SISO.

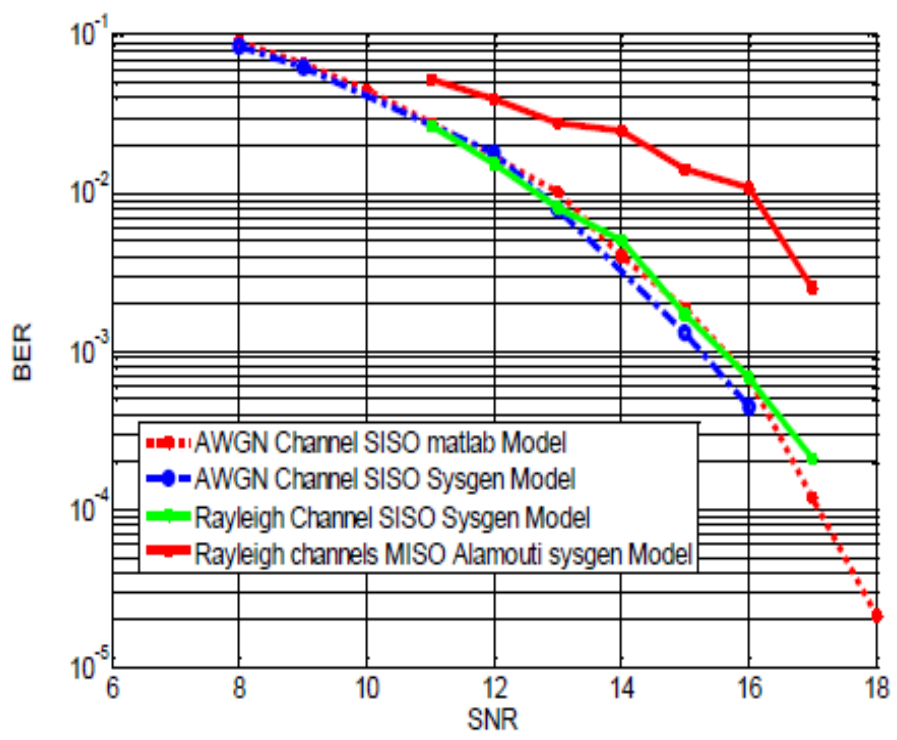

Fig. 8. SNR Vs BER for MISO, SISO

MISO system simulation assumes Gaussian channel because two channels will improve the performance of the network.

\section{A. Hardware Platform}

We used 2 Xilinx boards to implement this set up. One board is for transmitter and other board is for receiver. Transmitter was connected via LPX and HPC interfaces. RF board has the features of modulator and demodulator, Digital to Analog Converter (DAC) and Analog to Digital Converter (ADC) and other basic modules. 
Parameters and their Specifications

Oscillator frequency $=7.9 \mathrm{MHz}$

Base band frequency $=15 \mathrm{MHz}$

Scheme $=$ QPSK

Separation between carriers $=18 \mathrm{KHz}$

Bandwidth $=10 \mathrm{MHz}$

FFT length $=1024$

\section{SIMULATION RESULTS}

We simulated Digital Signal Processor using system generator to validate the working of algorithm. For channel simulations between the transmitter and receiver, we used different lengths of cables and a power combiner. The following figure 9 shows the ADC output at receiver end.

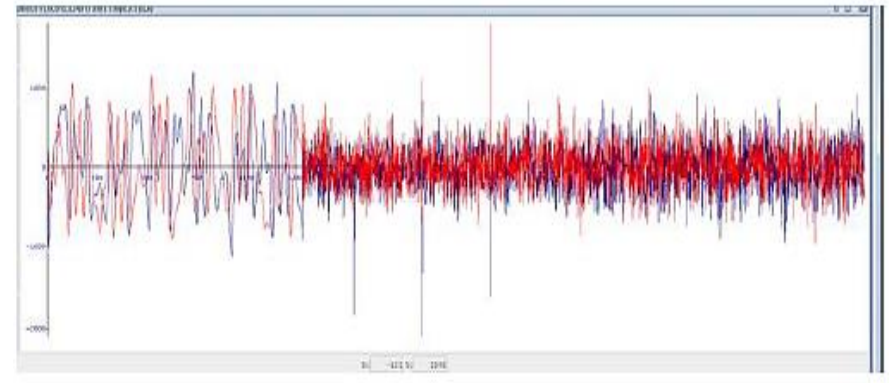

Fig. 9: ADC signal at the receiver input

This signal represents the received signal with the combination of two transmitting inputs. From figure 9, we can also observe that there are many peaks existed; this is due to frequency pilots.

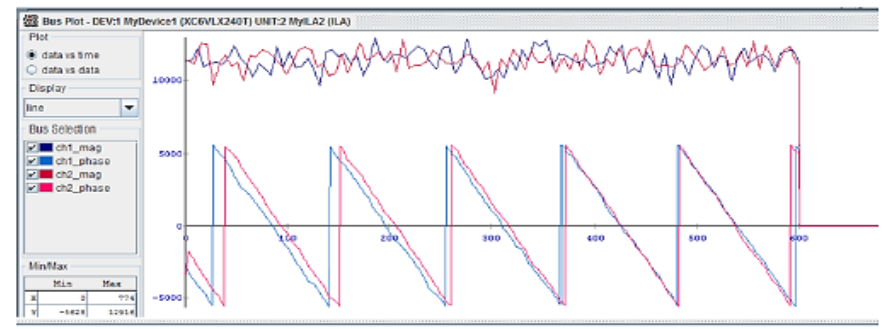

Fig. 10. OFDM symbol offset before compensation and after compensation

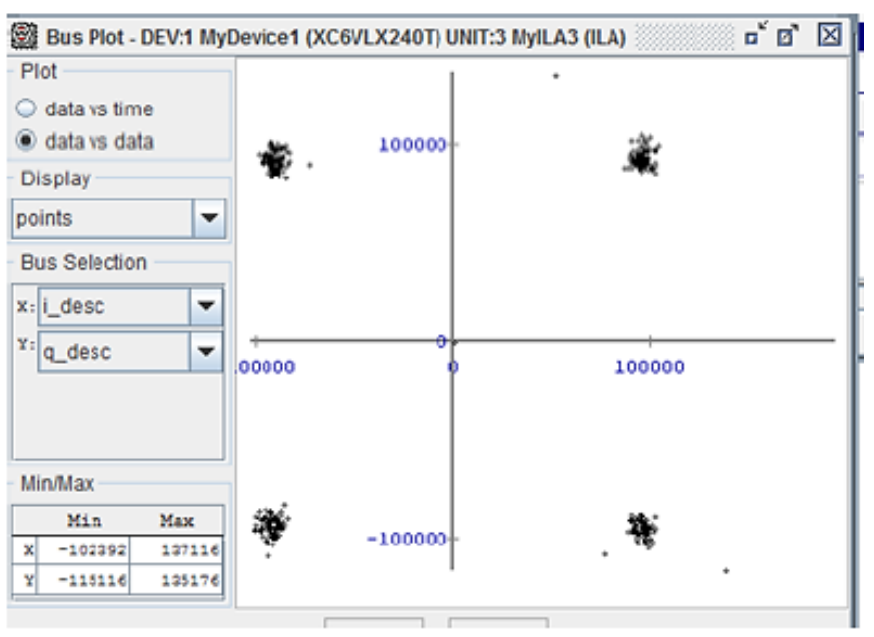

Fig. 11. QPSK constellation after decoding and equalization
The following figure 10 represents the estimated channel between antenna 1 and receiver and antenna 2 and receiver. It also shows about the phase and amplitude of the received signal. The simulation results are observed as flat frequency response curves with respect to magnitude and phase. The figure 11 shows the working of encoder and decoder. These results are shown the expected results which indicate the proper function of encoding and decoding blocks.

\section{CONCLUSION}

In this paper we presented Alamouti scheme implementation with the help of FPGA. The Alamouti scheme may simple for simulate but it requires enough attention to implement in a hardware platform. The real time test bed was implemented and analyzed with the help of Xilinx system generator for DSP. The simulation results showed that BER and SNR are considerably high for MISO than SISO. The results also proved that proposed OFDM based Alamouti implementation for MISO is excellent in all performance criterions

\section{REFERENCES}

[1] Seetaiah Kilaru, Aditya Gali,"Improving Quality of Service of Femto Cell Using Optimum Location Identification", IJCNIS, vol.7, no.10, pp.35-41, 2015.DOI: 10.5815/ijcnis.2015.10.04

[2] T. Pereira; Violas, M.; J.L. Lourenço; Gameiro, A.; Silva, A. ; Ribeiro, C.; "An FPGA Implementation of OFDM Transceiver for LTE Applications", Intrnl. Journal On Advances in Systems and Measurements, Vol. 6, No. 1-2, pp. 224 - 234, June, 2013.

[3] S. Kaiser, P. Hoeher, "Performance of multi-carrier CDMA systems with channel estimation in two dimensions," in Proc. IEEE Personal, Indoor and Mobile Radio Communications Symposium, pp. 115-119, Helsinki, Finland, September 1997

[4] P. Murphy, A. Sabharwal, and B. Aazhang, "On building a cooperative communication system: testbed implementation and first results", EURASIP Journal on Wireless Communications and Networking, June 2009, doi:10.1155/2009/972739

[5] Stefan Kaiser, "Space Frequency Block Coding and Code Division Multiplexing in OFDM Systems", IEEE Proceedings of GLOBECOM, 2003.

[6] J. Rinne, M. Renfors, "Pilot spacing in Orthogonal Frequency Division Multiplexing systems on practical channels," IEEE Transactions on Consumer Electronics, vol. 42 no. 3, pp. 959 - 962, November 1996

[7] P. Moose, "A technique for Orthogonal Frequency Division Multiplexing frequency offset correction", IEEE Transactions on Communications, vol. 42 no. 10, pp. 2908-2914, October 1984.

[8] Hui Liu and Guoqing Li, OFDM-Based Broadband Wireless Networks, John Wiley \& Sons, Inc., 2005

[9] P. Hoeher, S. Kaiser, P. Robertson, "Two-dimensional pilot-symbolaided channel estimation by Wiener filtering," in Proceedings of IEEE International Conference on Acoustics, Speech, and Signal Processing, pp. 1845-1848, April 199

[10] Jan-Jaap van de Beek, M. Sandell, and P. O. Börjesson, "ML estimation of time and frequency offset in OFDM systems", IEEE Transactions on Signal Processing, vol. 45, no. 7, July 1997

[11] A. Chini, "Multicarrier modulation in frequency selective fading channels," Ph.D. dissertation, Carleton University, Canada, 1994.

[12] M. Majó, "Design and implementation of an OFDM-based communication system for the GNU radio platform", Master Thesis, Dec. 2009.

[13] A. Dowler, A. Doufexi, A. Nix, "Performance Evaluation of Channel Estimation Techniques for a Mobile Fourth Generation Wide Area OFDM System,' in Proc. IEEE Vehicular Technology Conference, vol. 4, pp. 2036 - 2040, Vancouver, Canada, Sept. 2002.

[14] Albert A. Lysko, David L. Johnson," A Study of Propagation Effects in a Wireless Test Bed", in WSEAS Transactions on Communications, Issue 8, Volume 7, August 2008, pp.857-871

[15] 3GPP TS 36.201 V8.1.0, 3rd Generation Partnership Project; Technical Specification Group Radio Access Network; Evolved Universal Terrestrial Radio Access (E-UTRA);LTE Physical Layer - General Description, Nov. 2007. 
[16] S. Coleri, M. Ergen, A. Puri, A. Bahai, "Channel Estimation Techniques Based on Pilot Arrangement in OFDM Systems," IEEE Transactions on Broadcasting, vol. 48, no. 3, pp. 223-229, Sept. 2002.

[17] T.M. Schmidl and Cox, and D. C. Cox, "Robust frequency and timing synchronization for OFDM", IEEE Transactions on Communications, vol. 45, pp. 1613-1621, December 1997

[18] A. Marwanto, M. A. Sarijari, N. Fisal, S. K. S. Yusof, and R. A. Rashid, "Experimental study of OFDM implementation utilizing GNU Radio and USRP - SDR", Proc. of the IEEE 9th Malaysia International Conference on Communicatons, Dec. 2009, pp. 132-135.

[19] J. Garcia and R. Cumplido, "On the design of an FPGA-based OFDM modulator for IEEE 802.16-2004", 2005 International Conference on Reconfigurable Computing and FPGAs, 2005, pp. 22-25

[20] Y. Li, "Pilot-symbol-aided channel estimation for OFDM in wireless systems," IEEE Transactions on Vehicular Technology, Vol. 49, Issue 4, pp.1207-1215, July 2000

[21] G.J. Foschini and M.J. Gans, "On limits of wireless communications in a fading environment when using multiple antennas", Wireless Personal Communications Magazine, vol. 6, no. 3, Mar. 1998

[22] Kilaru, S., Harikishore, K., Sravani, T., Anvesh, C. L., \& Balaji, T. (2014, August). Review and analysis of promising technologies with respect to Fifth generation networks. In Networks \& Soft Computing (ICNSC), 2014 First International Conference on (pp. 248-251). IEEE.

[23] R. Negi, and J. Cioffi, "Pilot tone selection for channel estimation in a mobile OFDM system", Journal IEEE Transactions on Consumer Electronics, pp. 1122-1128, vol. 44 issue 3, August 1998, doi $10.1109 / 30.713244$

[24] Kilaru, S. (2014). Ability of OFDMA in Handling Interference of Femto Cells Under Random Access Process. Journal of Engineering Science and Technology Review, 7(2), 133-136.
[25] S. Boumard, A. Mammela, "Channel Estimation Versus Equalization in an OFDM WLAN System," in Proc. IEEE Vehicular Technology Conference, vol. 1, pp. 653-657, Rhodes, Greece, May 2001

[26] Kilaru, S., Prasad, Y. A., Kiran, K. S., \& Chandra, N. S. (2014). Design and Analysis of Heterogenious Networks. International Journal of Applied Engineering Research, 9(17), 4201-4208

[27] S. Syed Ameer Abbas, S. J. Thiruvengadam," Fpga Implementation Of 33GPP-Lte Physical Downlinkcontrol Channel Using Diversity Techniques", in WSEAS Transactions On Signal Processing, Issue 2 Volume 9, April 2013, pp 84-97

[28] C. Athaudage, A. Jayalath, "Low-Complexity Channel Estimation for Wireless OFDM Systems," in Proc. IEEE International Symposium on Personal, Indoor and Mobile Radio Communications, vol. 1, pp. 521 525, Beijing, China, Sept. 2003

[29] Kilaru, S. (2013, November). Public Safety Communication using Relay Node in LTE-Advanced Technology. In International Journal of Engineering Research and Technology (Vol. 2, No. 11 (November2013)). ESRSA Publications

[30] M. Shin, H. Lee, C. Lee, "Enhanced Channel Estimation Technique for MIMO-OFDM Systems," IEEE Transactions on Vehicular Technology, vol. 53, no. 1, pp. 261-265, Jan. 2004.

[31] J. Garcia and R. Cumplido, "On the design of an FPGA-based OFDM modulator for IEEE 802.11a”, 2nd International Conference on Electrical and Electronics Engineering, Sept. 2005, pp. 114-117.

[32] Anou Abderrahmane, Mehdi Merouane, Bensebti Messaoud, "Diversity Techniques to combat fading in WiMAX", in WSEAS Transactions on Communications, Issue 2, Volume 7, February 2008, pp.43-51. 